\title{
The influence of fine-needle aspiration biopsy of the thyroid gland on the size of the examined nodule and its ultrasound image
}

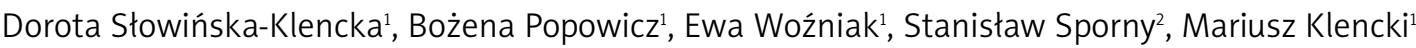

1Department of Morphometry of Endocrine Glands, Chair of Endocrinology, Medical University of Lodz, Poland

2Department of Dental Pathomorphology, Chair of Pathomorphology,

Medical University of Lodz, Poland

Submitted: 13 October 2010

Accepted: 31 January 2011

Arch Med Sci 2012; 8, 6: 1059-1064

DOI: 10.5114/aoms.2012.32415

Copyright @ 2012 Termedia \& Banach

\author{
Corresponding author: \\ Dorota Słowińska-Klencka \\ $\mathrm{MD}, \mathrm{PhD}$ \\ Department of Morphometry \\ of Endocrine Glands \\ Chair of Endocrinology \\ Medical University of Lodz \\ 5 Sterling St \\ 91-425 Lodz, Poland \\ Phone: +48 426324856 \\ E-mail: dsk@tyreo.am.lodz.pl
}

\begin{abstract}
Introduction: The aim of the study was to assess the influence of thyroid fineneedle aspiration biopsy (FNAB) on the size and ultrasound (US) features of the lesions and to examine whether the possible effects are persistent.

Material and methods: One hundred and fifty biopsied and 50 control thyroid nodules underwent two US examinations, 10-20 days and 8-10 weeks after the biopsy. The study took into account lesion volume alterations, both absolute and relative (with reference to its initial value), and the presence of US features of malignancy: hypoechogenicity, microcalcifications, internal blood flow, irregular or blurred margins and suspicious shape of the lesions. The analysis covered only those nodules which immediately after FNAB did not change their appearance owing to cyst fluid evacuation or intranodular hemorrhage.

Results: The increase of the lesion volume was more frequent in the group of biopsied lesions than the control one $(58.0 \%$ vs. $24.0 \%, p<0.0001)$ with the highest increase of $61.5 \%$. The mean change percentage, however, was determined below $5 \%$ and a tendency of the lesions to resume their initial volume was noticeable. Neither a persistent increase in nodule volume of above $50 \%$ nor significant changes in the presence of malignancy suggestive US features were observed after FNAB. None of the biopsied nodules developed any microcalcifications, irregular or blurred margins, internal blood flow, or suspicious shape.

Conclusions: Fine-needle aspiration biopsy does not cause permanent changes in the US image of biopsied lesions, provided that the sampling technique is appropriate and there are no significant changes observed during the biopsy.
\end{abstract}

Key words: thyroid, changes, fine-needle aspiration biopsy, follow-up.

\section{Introduction}

Fine-needle aspiration biopsy (FNAB) is the main method used in the preoperative diagnosis of thyroid nodules. Cytological outcome is of key importance in the selection of optimal treatment. The decision on performing FNAB is based mainly on the assessment of certain nodule features disclosed by ultrasound imaging (US). The following features are regarded as indicating suspicious nodules: hypoechogenicity, presence of microcalcification, irregular or microlobulated margin, taller-than-wide 
shape, and intranodular vascularity [1, 2]. These features have various predictive values in estimation of the risk of cancer in a nodule but when applied in combination they allow one to make the necessary selection of thyroid lesions for FNAB. It is of particular importance in endemic areas where the incidence of multinodular goiter is high [3]. Repeat (control) FNAB, after the first examination has detected a benign lesion, is mainly recommended in cases of nodules that have increased in size or developed any suspicious US features not present at the time of the first biopsy [1, 2]. The important question of what level of increase in the size of the lesion is significant still has not been settled. Nor has it been analyzed whether FNAB itself can change any of the US features, and if so, whether such changes are reversible.

The aim of the study was to assess the influence of FNAB on the size of the biopsied thyroid lesions and their features on ultrasound imaging as well as the persistence of possible effects.

\section{Material and methods}

The study included 100 consecutive euthyroid patients referred for FNAB for the first time. All the patients gave their informed consent to undergo two control US examinations: 10-20 days and 8-10 weeks after the biopsy. Those examinations allowed assessment of the US features of the biopsied lesions and to compare their values with those observed at the time of the biopsy. The examined features included the absolute and relative changes in the volume of the lesions with respect to their initial value. The volume of the lesions was calculated using a simplified elliptical shape volume formula: $1 / 2 \times$ width $\times$ depth $\times$ length of the measured structure. The presence of ultrasound features of malignancy in the biopsied lesions, such as hypoechogenicity, microcalcifications, internal blood flow, irregular or blurred margins, and shape index (defined as the ratio of the anterior-posterior dimension to the transverse dimension of a lesion, with values of the ratio $\geq 1$ regarded as positive), was also examined. The changes in those features during the observation were analyzed with respect to the initial classification of biopsied lesions into 3 size categories - lesions with the maximum diameter $\leq 10 \mathrm{~mm}$, in the range of $11-20 \mathrm{~mm}$, and $>20 \mathrm{~mm}$ - as well as with respect to the category of cytological outcome.

The patients had not previously been treated surgically or with ${ }^{131}$ | for thyroid diseases. No patients received any pharmacological treatment of the thyroid gland during the study. Patients receiving anticoagulants (therapeutic doses of acenocoumarol, warfarin or heparin) were excluded from the study. The analysis included only solid or mixed (solid-cystic) lesions with the cystic part smaller than $25 \%$ of the total lesion volume. Lesions from which more than $0.5 \mathrm{ml}$ of fluid was aspirated during FNAB were excluded from the analysis. By the same token, lesions significantly enlarged during FNAB (as a result of intranodular hemorrhage) were to be excluded. However, no such case was observed. The analysis did not include patients whose cytological outcomes indicated a need for rapid surgery and those who did not report for the control US examination. In total, the analysis covered 87 patients, including seven males. The mean age of the examined patients was $48.7 \pm 11.9$ years (mean \pm SD).

The same protocol was applied to the control group: 30 patients (including 2 males) of comparable age $(50.7 \pm 15.2$ years) with focal lesions in the thyroid. They did not undergo FNAB at the time but fulfilled the other inclusion criteria.

The US examinations were performed by 2 physicians with long experience in thyroid ultrasonography. The earlier studies showed high mutual reproducibility of the US measurements carried out by the two physicians. The US examinations were performed with the same equipment, namely Elegra Advanced (Siemens Medical Systems Inc., Issaquah, WA, USA) with a $7.5 \mathrm{MHz}$ linear transducer and power Doppler capability.

All the biopsies were US guided. In order to obtain a sufficient amount of diagnostic material, two aspirations of each examined lesion were usually performed. Ten milliliter syringes mounted in aspirating syringe holders with 25 gauge $(0.5 \mathrm{~mm}$ or $0.42 \mathrm{~mm}$ ) needles were used in the process. All smears were fixed in 95\% ethanol and stained with hematoxylin and eosin. The methods of classifying FNAB outcomes into categories were based on the guidelines formulated in the Bethesda system for reporting thyroid cytopathology [4].

Continuous variables were analyzed with Student's $t$-test. The comparison of frequency distributions was performed with $\chi^{2}$ test. The value of 0.05 was assumed to be the level of significance.

The study was approved by the ethics committee of the Medical University of Lodz.

\section{Results}

In total, 150 biopsied thyroid lesions (in 30 patients a single lesion was observed, in 51 patients 2 lesions, and in the other 6 patients 3 lesions) and 50 control lesions (in 12 patients a single lesion was observed, in 16 patients 2 lesions, and in 2 patients 3 lesions) were examined.

No statistically significant differences were found in the mean volumes of the thyroid glands between the patients subjected to FNAB and the control group $\left(22.53 \pm 11.44 \mathrm{~cm}^{3}\right.$ vs. $\left.19.85 \pm 10.92 \mathrm{~cm}^{3}\right)$ at the inclusion in the study. There were no significant differences in the features of the examined lesions between those groups at that time, including 
the mean volume $\left(2.13 \pm 3.11 \mathrm{~cm}^{3}\right.$ vs. $\left.1.94 \pm 2.48 \mathrm{~cm}^{3}\right)$, size distribution, and frequency of US features suggestive of malignancy (Table I). The echostructure of the biopsied lesions and the non-biopsied ones was also similar - solid nodules: 109 (72.7\%) vs. 30 (60.0\%), mixed nodules: 24 (16.0\%) vs. 12 (24.0\%), spongiform nodules: 17 (11.3\%) vs. 8 (16.0\%).

The control US examinations showed no significant changes in the mean thyroid volume nor in the mean volume of the examined lesions (Table II). However, the mean percentage change in the volume of the biopsied lesions was higher than in non-biopsied ones ( $4.5 \pm 15.9 \%$ vs. $-1.6 \pm 10.1 \%$, $p<0.01) 10-20$ days after FNAB. The highest increase was $61.5 \%$ in the FNAB group and $35.0 \%$ in the control group; the highest decrease was $-33.9 \%$ in the FNAB group and $-20.0 \%$ in the control group. In the group of biopsied lesions, an increase in the volume of the lesions was observed more frequently than in the control group $(58.0 \%$ vs. $24.0 \%$, $p<0.0001)$, and a decrease in the volume was less frequent $(31.3 \%$ vs. $52.0 \%, p<0.02)$.

At least $20 \%$ increase in the volume was observed more frequently in the group of biopsied lesions than in the control group $(26 / 17.3 \%$ vs. $3 / 6.0 \%, p<0.05$ ) (Table III). There was no significant difference between the examined groups in the percentage of lesions that decreased by more than $20 \%$ in volume $(11 / 7.3 \%$ vs. $2 / 4.0 \%$, NS).

An increase in volume of at least $50 \%$ in the first control US examination was noted in two cases of biopsied lesions (1.3\%) but not in the control group. In both those cases at the time of the second control US examination (8-10 weeks after FNAB) the lesions were $40 \%$ larger than initially. In one case of a non-biopsied lesion (2.0\%) $50 \%$ or higher increase in its volume was observed only in the second control US examination. In all 3 cases where the increase in volume exceeded $50 \%$ of the initial value, the maximal diameters of the lesions were below $15 \mathrm{~mm}$, and the lesions did not satisfy the commonly used criterion of simultaneous enlargement of at least two dimensions by at least $2 \mathrm{~mm}$ (Table III).

In the US examination performed 8-10 weeks after FNAB no statistically significant differences were observed in the percentage of volume changes between biopsied and non-biopsies lesions, nor in

Table I. Ultrasound characteristics of analyzed lesions

\begin{tabular}{|lcccccc|}
\hline Suspicious ultrasound features & \multicolumn{5}{c|}{ Number/\% of nodules in size class } \\
\cline { 2 - 7 } & \multicolumn{3}{c|}{ FNAB group } & \multicolumn{3}{c|}{ Control group } \\
\cline { 2 - 7 } & $\leq 10 \mathrm{~mm}$ & $11-20 \mathrm{~mm}$ & $>20 \mathrm{~mm}$ & $\leq 10 \mathrm{~mm}$ & $11-20 \mathrm{~mm}$ & $>20 \mathrm{~mm}$ \\
\hline Marked hypoechogenecity & $17 / 47.2$ & $28 / 37.8$ & $9 / 22.5$ & $6 / 37.5$ & $5 / 23.8$ & $3 / 23.1$ \\
\hline Internal blood flow & $14 / 38.9$ & $27 / 36.5$ & $23 / 57.5$ & $4 / 25.0$ & $8 / 38.1$ & $5 / 38.5$ \\
\hline Irregular or blurred margins & $2 / 5.5$ & $3 / 4.1$ & $2 / 5.0$ & $1 / 6.2$ & $2 / 9.5$ & $1 / 7.7$ \\
\hline Taller than-wide shape & $6 / 16.7$ & $13 / 17.6$ & $2 / 5.0$ & $3 / 18.7$ & $4 / 14.2$ & $0 / 0.0$ \\
\hline Microcalcifications & $2 / 5.5$ & $3 / 4.1$ & $2 / 5.0$ & $0 / 0.0$ & $0 / 0.0$ & $1 / 7.7$ \\
\hline Number/\% of all nodules in group & $36 / 24.0$ & $74 / 49.3$ & $40 / 26.7$ & $16 / 32.0$ & $21 / 42.0$ & $13 / 26.0$ \\
\hline
\end{tabular}

Table II. The changes of the thyroid volume and the lesions volume in control US examinations

\begin{tabular}{|llccc|}
\hline Feature & Group & \multicolumn{3}{c|}{ Time of examination } \\
\cline { 2 - 5 } & & At the time of FNAB & 10-20 days after FNAB & 8-10 weeks after FNAB \\
\hline Mean thyroid volume $\left[\mathrm{cm}^{3}\right]$ & FNAB & 22.53 & 21.85 & 21.99 \\
\cline { 2 - 5 } & Control & 19.85 & 20.24 & 19.64 \\
\hline Mean lesion volume $\left[\mathrm{cm}^{3}\right]$ & FNAB & 2.13 & 2.20 & 2.15 \\
\cline { 2 - 5 } & Control & 1.94 & 1.87 & 1.88 \\
\hline $\begin{array}{l}\text { Number/\% of lesions which } \\
\text { enlarged }\end{array}$ & FNAB & - & $87 / 58.0 \%{ }^{* *}$ & $78 / 52.0 \%$ \\
\cline { 2 - 5 } $\begin{array}{l}\text { Number/\% of lesions which } \\
\text { diminished }\end{array}$ & Control & - & $12 / 24.0 \%$ & $20 / 40.0 \%$ \\
\cline { 2 - 5 } $\begin{array}{l}\text { Mean percentage change } \\
\text { of lesion volume in with } \\
\text { respect to the initial value }\left[\mathrm{cm}^{3}\right]\end{array}$ & Control & - & $47 / 31.3 \%{ }^{* * *}$ & $58 / 38.7 \%$ \\
\cline { 2 - 5 } & Control & - & $26 / 52.0 \%$ & $25 / 50.0 \%{ }^{*}$ \\
\hline
\end{tabular}

${ }^{*} p<0.01$ vs. 8-10 weeks after FNAB, ${ }^{* *} p<0.0001$ vs. control, ${ }^{* * *} p<0.02$ vs. control 
the number of lesions that changed in volume (Tables II and III). The biopsied lesions tended to decrease in the mean percentage of volume change with respect to the initial value between the first and the second control examination ( $4.5 \pm 15.9 \%$ vs. $1.7 \pm 14.6 \%$, NS).

The characteristics of the US features useful in selection of thyroid lesions for biopsy did not change significantly after FNAB. None of the examined lesions showed any change related to the borders of the lesion or the presence of microcalcifications. One lesion in the control group and 2 biopsied lesions became transiently hypoechoic (in the first control US examination only). In 1 patient of the group subjected to FNAB a lesion isoechoic at the time of biopsy was found to be hypoechoic in the second control US examination. As to the shape index, it was found that 2 out of 21 (9.5\%) biopsied lesions (one of diameter $\leq 10 \mathrm{~mm}$ and another with diameter in the range of $11-20 \mathrm{~mm}$ ) and 1 out of 7 (14.3\%) non-biopsied ones (of diameter $\leq 10 \mathrm{~mm}$ ) that were positive at the time of FNAB turned out to be negative in the first control US examination. Two of them (one of each group) became again positive in the second control US examination. In all those cases the observed change in the shape index was a consequence of a $1 \mathrm{~mm}$ change in a dimension of the lesion. In 2 out of 64 (3.1\%) biopsied lesions (both of diameter $\leq 10 \mathrm{~mm}$ ) and in 1 out of $17(5.9 \%)$ control lesions (of diameter in the range of $11-20 \mathrm{~mm}$ ) an internal pattern of vascularization was found at the time of FNAB that was not observed any longer in the control examinations. In none of the examined lesions was any change in echostructure observed.

The outcomes of FNAB examinations are shown in Table IV. No relation was found between the cytological outcome and the percentage of lesion enlargements after FNAB. In all the lesions that enlarged by at least $50 \%$ the cytological outcome was categorized as benign nodules. The percentage of cytological outcomes classified as "suspicious for follicular neoplasm" in the group of lesions that enlarged by $20 \%$ or more and in the group of other lesions was the same.

\section{Discussion}

This study constitutes one of the first prospective evaluations of the possible influence of FNAB on US features of thyroid nodules which are considered when diagnostic and therapeutic decisions are made. Some previous prospective studies only analyzed the influence of FNAB on size of lesions $[5,6]$. In ret-

Table III. Number and percentage of lesions which changed volume in control US examinations by $\geq 20 \%$ and by $\geq 50 \%$ in with respect to the initial value

\begin{tabular}{|c|c|c|c|c|c|c|c|c|}
\hline \multirow[t]{4}{*}{ Group } & \multicolumn{8}{|c|}{ Number/\% of lesions that changed volume in the control examinations } \\
\hline & \multicolumn{4}{|c|}{ 10-20 days after FNAB } & \multicolumn{4}{|c|}{ 8-10 weeks after FNAB } \\
\hline & \multicolumn{2}{|c|}{$\geq 20 \%$ change } & \multicolumn{2}{|c|}{$\geq 50 \%$ change } & \multicolumn{2}{|c|}{$\geq 20 \%$ change } & \multicolumn{2}{|c|}{$\geq 50 \%$ change } \\
\hline & Increase & Decrease & Increase & Decrease & Increase & Decrease & Increase & Decrease \\
\hline FNAB & $26 / 17.3^{*}$ & $11 / 7.3$ & $2 / 1.3$ & $0 / 0.0$ & $10 / 6.7^{a}$ & $10 / 6.7^{b}$ & $0 / 0.0$ & $0 / 0.0$ \\
\hline Control & $3 / 6.0$ & $2 / 4.0$ & $0 / 0.0$ & $0 / 0.0$ & $4 / 8.0^{c}$ & $3 / 6.0^{c}$ & $1 / 2.0$ & $0 / 0.0$ \\
\hline
\end{tabular}

a 5 cases the volume increase $\geq 20 \%$ persisted from the first control examination and in 5 other cases it was observed only on the second control US, $b_{\text {in }} 4$ cases the volume decrease $\geq 20 \%$ persisted from the first control examination and in 6 other cases it was observed only on the second control US, "the volume change by $\geq 20 \%$ was observed only on the second control US; ${ }^{*} p<0.05 \mathrm{vs}$. control

Table IV. The categorization of fine-needle aspiration biopsy (FNAB) results

\begin{tabular}{|c|c|c|}
\hline Cytological results & $\begin{array}{l}\text { Categories of } \\
\text { cytological outcomes }\end{array}$ & $\begin{array}{l}\text { Number, } \\
\% \text { of FNABs }\end{array}$ \\
\hline $\begin{array}{l}\text { Thyroid follicular cells without or with mild anicocytosis anisocytosis } \\
\text { or admixture of follicular cells and Hürthle cells in a background } \\
\text { of abundant colloid and macrophages }\end{array}$ & Benign lesions & $119,79.3$ \\
\hline $\begin{array}{l}\text { Scant colloid, Hürthle cells, follicular cells, lymphocytes seen in the background } \\
\text { percolating between cell groups - chronic thyreoiditis }\end{array}$ & & $13,8.7$ \\
\hline $\begin{array}{l}\text { Follicular cells arranged in cohesive groups and microfollicles with focal nuclear } \\
\text { crowding and overlapping and monolayer sheets in a background of watery } \\
\text { colloid admixed with thick colloid and few macrophages }\end{array}$ & $\begin{array}{l}\text { Follicular lesions } \\
\text { of undetermined } \\
\text { significance }\end{array}$ & $8,5.3$ \\
\hline $\begin{array}{l}\text { Monomorphic follicular cells usually arranged in three three-dimensional groups } \\
\text { and microfollicles with prominent nuclear overlapping and crowding, minimal } \\
\text { or absent background colloid }\end{array}$ & $\begin{array}{l}\text { Suspicious for } \\
\text { follicular neoplasm }\end{array}$ & $8,5.3$ \\
\hline Acellular specimen & Non-diagnostic & $6,4.0$ \\
\hline
\end{tabular}


rospective studies, the changes of the size of lesions and in their structure were described as a consequence of intranodular hemorrhage or an enlargement of the thyroid gland resulting from hematoma or transient swelling [7]. Those studies did not evaluate the influence of biopsy free from clinical complications on US features of thyroid lesions.

Our data indicate that the FNAB procedure itself does not significantly influence the US features that are useful in the selection of thyroid lesions for cytological examination. The changes of size of biopsied lesions - though statistically higher than in the control group - are transient and their degree is not relevant clinically. Currently, a clinically significant increase in the size of lesions is assumed to be at least $50 \%$ of the initial volume, or $20 \%$ in the case of the nodule diameter, with a minimum $2 \mathrm{~mm}$ increment in two or more dimensions $[2,8,9]$. The latter condition is particularly important in the case of small lesions, when the effect of accuracy of measurements on calculated volume is relatively high. In our data, $50 \%$ or higher increase in volume was observed only in lesions with the maximal diameter $\leq 15 \mathrm{~mm}$. In none of those cases was the $2 \mathrm{~mm}$ minimal increase in two or more dimensions observed. The tendency of the lesions to resume their initial sizes is also worth noticing. It is consistent with the findings of Gordon et al. that higher variation in nodule size is observed in the examination performed 1 month after FNAB than 6 months after biopsy. They also observed a tendency for nodule volume to decrease during follow-up after FNAB [5]. That study was based on a considerably smaller number of lesions with their mean volume being several times higher than ours. The influence of FNAB on the dimensions of thyroid lesions of similar size was assessed by Güney et al. [6]. The authors did not find any difference in mean volume of nodules before and after FNAB, which is in agreement with our data. However, their study included many lesions which immediately after FNAB changed in volume by more than $50 \%$ (increased or decreased). The authors reasonably concluded that that could be the reason for the lack of statistically significant differences in mean volumes observed at the following time points. In our study such lesions were excluded as we believe that all noticeable changes in the volume of a biopsied lesion should be described in the cytological report. A similar conclusion was formulated by Güney et al. They suggested that nodule volume shortly after FNAB, and not before FNAB, should be considered during the follow-up [6]. Apart from the size of lesions, both mentioned studies did not evaluate the other features.

Our results did not reveal any significant influence of FNAB on the values of US features suggestive of malignancy. The isolated cases of obser- ved changes in these features were related to hypoechogenicity, the internal pattern of vascularization or the 'shape' criterion. Those cases included lesions from both the FNAB and the control groups. It probably reflects difficulty with reproducible measurement of US features rather than the influence of FNAB [10]. According to Choi et al. the reproducible assessment of hypoechogenicity is the most difficult [10]. When assessing the shape of a lesion, lack of accuracy in the measurements is particularly important in cases in which both analyzed dimensions (anterior-posterior and width) are very similar. The intensified internal vascular flow that was observed in several cases at the biopsy, but absent in the control US examination, could possibly be the result of changes in blood pressure. In no biopsied lesions did persistent microcalcifications, blurred margins, internal pattern of blood flow or taller-than-wide shape occur after FNAB. Only in one case of control examination 8-10 weeks after FNAB was a lesion described earlier as isoechoic found to be hypoechoic.

It should be stressed that our conclusions are related to solid lesions or mixed, mainly solid lesions (> $75 \%$ of total volume) in which no significant changes in US image were observed during FNAB. The obtained results are undoubtedly affected by the particular FNAB procedure, which at our center is usually performed by 2 punctures with a 25 gauge $(0.5 \mathrm{~mm}$ or $0.42 \mathrm{~mm})$ needle, and the reduced pressure is adjusted according to the relative ease of obtaining material from the lesion. Immediately after the biopsy, brief (for 1-2 min) pressure at the puncture site is usually applied. The anticoagulant treatment, coagulopathy, higher number of punctures, the use of thicker needles or rough sampling technique may all significantly influence the size and the structure of the lesion at the time of FNAB or with some delay.

In conclusion, ultrasound imaging is an important procedure in the follow-up of patients with focal lesions in the thyroid gland. A significant increase of lesion size, and occurrence of features suggestive of malignancy are among relevant factors suggesting the necessity of control FNAB or even of surgical treatment. Adequate clinical interpretation of the observed changes in the US image of examined lesions requires information on all diagnostic or therapeutic procedures which were performed between control examinations. In most cases FNAB does not cause permanent changes in the US image of biopsied lesions, provided that there are no significant changes observed during the biopsy and the sampling technique is appropriate.

\section{Acknowledgments}

The study was supported financially by grant no. 502-11-571 from the Medical University of Lodz. 


\section{References}

1. American Association of Clinical Endocrinologists, Associazione Medici Endocrinologi, and European Thyroid Association medical guidelines for clinical practice for the diagnosis and management of thyroid nodules. Gharib H, Papini E, Paschke R, et al. AACE/AME/ETA Task Force on Thyroid Nodules. Endocr Pract 2010; 16 (Suppl 1): 1-43.

2. Cooper DS, Doherty GM, Haugen BR. Revised American Thyroid Association Management Guidelines for patients with thyroid nodules and differentiated thyroid cancer. Thyroid 2009; 19: 1167-214.

3. Słowińska-Klencka D, Popowicz B, Lewiński A, Sporny S, Klencki $M$. The fine-needle aspiration biopsy efficacy of small thyroid nodules in the area of recently normalized iodine supply. Eur J Endocrinol 2008; 159: 747-54.

4. The Bethesda system for reporting thyroid cytopathology. Definitions, criteria and explanatory notes. Ali SZ, Cibas ES (eds.). $1^{\text {st }}$ ed., Springer, New York 2010.

5. Gordon DL, Flisak M, Fisher SG. Changes in thyroid nodule volume caused by fine-needle aspiration: a factor complicating the interpretation of the effect of thyrotropin suppression on nodule size. I Clin Endocrinol Metab 1999; 84: 4566-9.

6. Güney E, Ozgen AG, Kabalak T. Is the effect of fine-needle aspiration biopsy on the thyroid nodule volume important to evaluate the effectiveness of suppression therapy? J Endocrinol Invest 2003; 26: 651-4.

7. Polyzos SA, Anastasilakis AD. Clinical complications following thyroid fine-needle biopsy: a systematic review. Clin Endocrinol 2009; 71: 157-65.

8. Brauer VF, Eder P, Miehle K, Wiesner TD, Hasenclever H, Paschke R. Interobserver variation for ultrasound determination of thyroid nodule volumes. Thyroid 2005; 15 : 1169-75.

9. Oertel YC, Miyahara-Felipe L, Mendoza MG, Yu K. Value of repeated fine needle aspirations of the thyroid: analysis of over ten thousand FNAs. Thyroid 2007; 17: 1061-6.

10. Choi SH, Kim EK, Kwak JY, Kim MJ, Son EJ. Interobserver and intraobserver variations in ultrasound assessment of thyroid nodules. Thyroid 2010; 20: 167-72. 